\title{
The Evaluation of the Effect of Community Opening on the Peripheral Road
}

\author{
Chenchen Huang \\ Department of Economics and Management, North China Electric Power University, \\ Baoding 071000, China \\ 973118497@qq.com
}

Keywords: analytic hierarchy process, open area, road access

\begin{abstract}
The effect of community opening may be related to residential structure, the surrounding road structure and traffic flow. After the opening of the community changed the road network structure and shared the surrounding road traffic pressure, which for the residential area and the city's traffic will have an impact. This paper aims at the impact of the open area on the surrounding road traffic. We used the Analytic Hierarchy Process (AHP) to analyze the 11 indicators in the evaluation system through the establishment of evaluation index system and obtained a number of factors which have a greater impact. We quantified these factors separately and found out the influential factors to study the impact of community opening on the surrounding road traffic.
\end{abstract}

\section{Introduction}

In February 21, 2016, the State Council issued "on the further strengthening of urban planning and construction management of a number of opinions". Among them, Article 16 of the views on the promotion of neighborhood system, has aroused wide attention and discussion. In this paper, a suitable evaluation index system is selected to evaluate the impact of community opening on the surrounding road traffic, which provides a quantitative basis for scientific decision-making of urban planning and traffic management departments, which has important practical significance.

\section{Problem Analysis}

The impact of community opening on road traffic is mainly reflected in road capacity. Different from the general evaluation system of road traffic, this paper focuses on the impact of open district. Among the many influencing indexes of road capacity, we should select the indexes which have great relevance and easy to get accurate data. In addition, we should consider the position and area of the cell's influence on the indicators .

\section{Road Traffic Evaluation Index System}

Open community is intended to break the city road congestion situation and ease traffic pressure. Road traffic evaluation index should be able to fully reflect the opening area's impact of the surrounding road traffic. While the performance indicators of district and traffic conditions can't be a separate reflection of the open area's impact on the surrounding road traffic. Therefore, we should consider the district, the surrounding traffic conditions and the surrounding road performance indicators. According to the principles ${ }^{[1]}$ that establishing index system with science, this paper presents the evaluation system shown in Figure 1. 


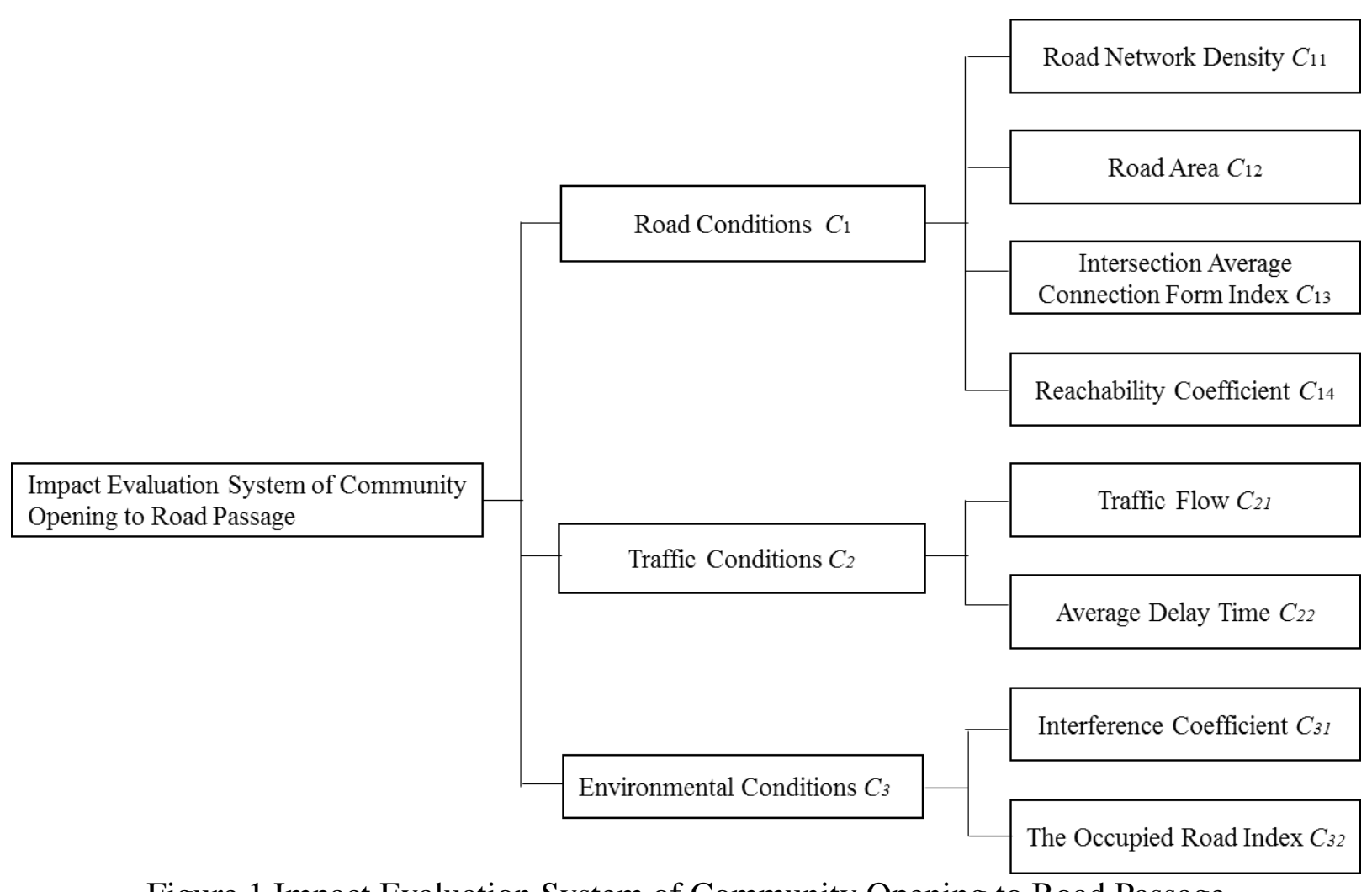

Figure 1 Impact Evaluation System of Community Opening to Road Passage

\section{Quantitative Evaluation of Indicators}

In the evaluation index system, some indicators are quantitative, some are qualitative, so we should do the qualitative analysis of quantitative indicators.

- Road network density $\left(\mathrm{km} / \mathrm{km}^{2}\right)$

It refers to the ratio of total length of urban roads and urban land area, the formula is

$$
\delta=\frac{\sum l_{i}}{S}
$$

Where $S$ is the urban construction land area $\left(\mathrm{km}^{2}\right) ; l_{i}$ is the length of each section of the city $(\mathrm{km})$; the greater the density of the road network, the higher the traffic capacity.

- Road Area $\left(m^{2}\right)$

The road area refers to the total area of urban traffic roads. It combined with road network density can reflect the average width of the road, the formula is

$$
S_{\mathrm{z}}=S_{0}+S_{i} \times k_{i}
$$

Where $S_{0}$ is the total area of the urban traffic road except the residential road; $S_{i}$ is the area of the residential area $i$; $k_{i}$ is the road area ratio of the planning and design standard of the residential area.

- Intersection average connection form index

Considering many non-motor vehicles go in or out of the district, the district is best to connect with the slip road while connect with the city expressway or trunk road directly is unreasonable. In this paper, the complexity of road conflict is described by means of the average connection between the community and surrounding intersections. Select the following four cases, given the appropriate value:

Residential road connected with each other ${ }^{[2]}$, it's value is 1 (which is only considered after opening);

The residential road is connected with the urban slip road, and its value is 2;

Community roads is connected with urban roads, it's value is 3; 
Residential road and urban expressway connected, its value is 4;

Intersection average connection form index is expressed as:

$$
Z=\frac{\sum_{i=1}^{4} n_{i} \times i}{\sum_{i=1}^{4} n_{i}}
$$

Where $n_{i}$ is the number of intersections corresponding to the case.

- Reachability Coefficient

Before and after the community opening has great influence on the reachability coefficient. The coefficient refers to the ratio of the total length of the main roads around the residential area to the sum of the shortest path between the center of the district and the surrounding roads. It describes the convenience of traveling to the trunk network in the district. It is very easy to be "poles apart" before the community opening. The expression is

$$
\alpha=\frac{\sum_{i} l_{i}}{\sum_{k=1}^{4} d_{k}}
$$

Where $d_{k}$ is the shortest path from the center of the district to the four directions and $l_{i}$ is the length of the main line of each section of the surrounding area.

- Traffic Flow ( $p c u / h$ )

It refers to the number of vehicles passed at a road point in a given period of time. The community opening have an impact on the total number of traffic outside the community, because the open community can share the peripheral branches of non-motor vehicle traffic, relieve road pressure. Therefore, this article reflect the traffic pressure through the average traffic volume outside the road sections of the district. This paper is simulated by traffic simulation software to get the data.

$$
\bar{v}=\frac{\sum v_{i}}{n}
$$

Where $n$ is the number of links which is directly connected to the community.

- Average delay time ( $s$ )

Although the opening of the community could ease the flow of traffic pressure, more cross-road also brought a longer delay time. The average delay time is expressed as:

$$
d=\frac{0.5 T\left(1-\frac{t_{g}}{T}\right)}{1-\left[\min (1, x) \cdot \frac{t_{g}}{T}\right]}
$$

Where $T$ is the measured or simulated non-motor vehicle traffic on the road. $Q_{b}$ is the capacity of non-motorized vehicles per meter on the road. $W_{1}$ is the width of the unidirectional lane, and $W_{2}$ is the width of the one-way lane.

- Interference coefficient

Community opening will increase the interference of the passage of non-motor vehicles such as bicycles, electric cars and pedestrian and affect the road capacity. This paper uses the interference coefficient to measure it. The expression is:

$$
\eta=0.8-\left(q_{b} / Q_{b}+0.5-W_{2}\right) / W_{1}
$$

$q_{b}$ is the measured or simulated non-motor vehicle traffic on the road; $Q_{b}$ is the non-motor vehicle capacity per meter on the road; $W_{1}$ is the one-way non-motorized lane width; $W_{2}$ is the one-way motorway width.

- The Occupied Road Index 
As the car has a higher volume, the community roads were occupied by vehicles and the community opening will inevitably lead to be more street vendors to increase road occupancy. This paper evaluates it through the ratio of non-flow occupied area and total traffic area.

$$
\beta=\frac{S_{m}}{S}
$$

$S_{m}$ is the area that community and the surrounding roads at a certain point of time occupied by non-traffic factor; $S$ is the total area of the community and the surrounding roads. Note: An unopened cell should be regarded as a closed area, which should not be calculated within the area of road traffic but only the surrounding of the community.

\section{Using AHP method to determine the index weight}

In this paper, AHP (Analytic Hierarchy Process) ${ }^{[3]}$ is used to determine the subjective weights of each index. We established a hierarchical structure of indicators. The judgment matrix and weight of the criterion layer are shown inTable 1:

Table 1 The first-level index judgment matrix and weight

\begin{tabular}{ccccc}
\hline$C$ & $C_{1}$ & $C_{2}$ & $C_{3}$ & Weights \\
\hline$C_{1}$ & 1 & 4 & 5 & 0.6833 \\
$C_{7}$ & $1 / 4$ & 1 & 2 & 0.1998 \\
$C_{3}$ & $1 / 5$ & $1 / 2$ & 1 & 0.1168 \\
\hline
\end{tabular}

The judgment matrix and weights of scheme layer are shown in Table 2, Table 3 and Table 4.

Table 2 The second-level judgment matrix and weight

\begin{tabular}{cccccc}
\hline$C_{1}$ & $C_{11}$ & $C_{12}$ & $C_{13}$ & $C_{14}$ & Weights \\
\hline$C_{11}$ & 1 & 5 & 2 & 3 & 0.4773 \\
$C_{11}$ & $1 / 5$ & 1 & $1 / 4$ & $1 / 2$ & 0.0809 \\
$C_{12}$ & $1 / 2$ & 4 & 1 & 2 & 0.2880 \\
$C_{11}$ & $1 / 3$ & 2 & $1 / 2$ & 1 & 0.1539 \\
\hline
\end{tabular}

Table 3 Judgment Matrix and Weight of Secondary Index

\begin{tabular}{cccc}
\hline$C_{2}$ & $C_{21}$ & $C_{22}$ & Weights \\
\hline$C_{21}$ & 1 & 2 & 0.6667 \\
$C_{2}$ & $1 /$ & 1 & 0.333 \\
\hline
\end{tabular}

Table 4 Two-level indicator judgment matrix and weight

\begin{tabular}{cccc}
\hline$C_{3}$ & $C_{31}$ & $C_{32}$ & Weights \\
\hline$C_{21}$ & 1 & 3 & 0.75 \\
$C_{32}$ & $1 / 3$ & 1 & 0.25 \\
\hline
\end{tabular}

The maximum eigenvalue of the judgment matrix is calculated by Matlab software: $\lambda_{\text {max }}=3.0246$,then the consistency index is calculated: $C I=0.0123$, and the correction coefficient is obtained by the lookup table: $R I=0.58$, and the consistency proportion is calculated: $C R=0.0212<0.10$, which is satisfy the consistency test. After the consistency check, we calculate the first-level index weight distribution as: $V=(0.6833,0.1998,0.1168)$.

In the same way, the judgment matrix and weight distribution of the second-order index are obtained, and the judgment matrix is not more than 0.1 , and the judgment matrix meets the consistency requirement. The specific weights of the indicators are as follows:

Road conditions: $V_{1}=(0.4773,0.0809,0.2880,0.1539)$; 
Traffic Conditions: $V_{2}=(0.6667,0.3333)$;

Environmental conditions: $V_{3}=(0.75,0.25)$;

Therefore, the corresponding weight set of eight indicators is

$$
W=(0.3261,0.0553,0.1968,0.1051,0.1332,0.0665,0.0876,0.0292)
$$

\section{Conclusions}

This paper selected the important indexes of road network density: the number of intersections, traffic volume and so on, and established the evaluation index system of the effect of community opening on surrounding road traffic. After the system was established, the qualitative indexes were quantified and the weight of each index was determined by AHP. Whether the openness of the community can improve the road capacity and improve the traffic conditions need further analysis and research.

\section{References}

[1] Zhao Q, Huang S M, Si-Xiang D I, et al. Comprehensive Evaluation Index System of Urban Public Traffic System[J]. Transport Standardization, 2009.

[2] Ding B Q, Song X Y. Traffic Blocking Evaluation Index System of Grade Intersection[J]. Transport Standardization, 2009.

[3] Cheng E W L, Li H, Ho D C K. Analytic hierarchy process (AHP)[M]// Encyclopedia of Biostatistics. John Wiley \& Sons, Ltd, 2013:19-28. 